\title{
The Remarkable Pliability and Promiscuity of Specialized Metabolism
}

\author{
J.-K. WENG AND J.P. NOEL \\ Howard Hughes Medical Institute, Jack H. Skirball Center for Chemical Biology \& Proteomics, \\ The Salk Institute for Biological Studies, La Jolla, California 92037 \\ Correspondence: noel@salk.edu
}

\begin{abstract}
Metabolic pathways are often considered "perfected" or at least predictable as substrates efficiently rearrange into products through the intervention of an optimized enzyme. Moreover, single catalytic steps link up, forming a myriad of metabolic circuits that are often modeled with a high degree of certainty. However, on closer examination, most enzymes are not precise with respect to their activity, using not just one substrate but often a variety and producing not just one product but a diversity. Hence, the metabolic systems assembled from enzymes possessing varying degrees of what can be termed catalytic promiscuity are not clear-cut and restrictive; rather, they may at times operate stochastically in the intracellular milieu. This "messiness" complicates our understanding of normal and aberrant cellular behavior, while paradoxically sowing the seeds for future advantageous metabolic adaptations for host organisms. Catalytic promiscuity is intrinsically associated with the dynamic nature of enzyme structures and their chemical mechanisms, both key to enzyme and metabolic evolvability. In addition to primary (core) metabolism, which is essential for survival, organisms also possess highly elaborated secondary (specialized) metabolic systems. These specialized enzymes and pathways often provide unique adaptive strategies for a myriad of organisms and their populations in challenging and changing ecosystems. Generally, enzymes of specialized metabolism show attenuated kinetic activities and expanded catalytic promiscuity compared with their phylogenetic relatives rooted in primary metabolism. We propose that evolvability may be a selected trait in many specialized metabolic systems spread across populations of organisms exposed to continually fluctuating biotic and abiotic environmental pressures. As minor metabolites arising from catalytic messiness provided enhanced population fitness, specificity relaxed, and catalytic efficiency was attenuated. This updated view provides a mechanistic basis for reaching a deeper understanding of the evolutionary underpinnings of the explosion of chemodiversity in nature.
\end{abstract}

Fundamentally, metabolism is a chemical property of living organisms, defined as the collection of enzymecatalyzed chemical reactions that synthesize or deconstruct metabolic chemicals necessary to sustain life and contribute to the reproductive success of host populations in the face of ever-changing environmental pressures. Similarly to other catalysts, enzymes do not alter the equilibrium of the chemical reactions but enhance the rate of the reactions by lowering their activation energies (Cook and Cleland 2007). Reactions catalyzed by enzymes are generally precise and efficient, involving specific metabolic substrates transformed into predictable metabolic products using defined catalytic mechanisms. This ordered view of one enzyme-one mechanism-one product comes from seminal discoveries made by many investigators during the last century, focused for excellent practical reasons on the phylogenetically conserved enzymes of primary metabolism.

Many primary metabolic enzymes retain a high degree of conserved function regardless of the complexity of the organismal host. They often participate in core metabolic reactions involving energy metabolism (e.g., fermentation and oxidative phosphorylation) or the biosynthesis of biological building blocks (e.g., proteins, nucleic acids, and carbohydrates) (Kühne 1877; Shevelev and Hubscher 2002). Moreover, although some degree of catalytic promiscuity is an inherent trait of nearly all enzymes, primary metabolic enzymes often possess such vanishingly low levels of promiscuity that until recently it was problematic to detect and quantify.

Elevated to a systems-level approach, this generalized view of primary metabolism projects a prevailing textbook depiction of metabolism as highly ordered networks of optimized, or at a minimum, predictable chemical reactions (Fig. 1A). Indeed, this apparent uniformity is a key assumption necessary for many mathematical models of primary metabolic systems, using computationally intensive systems-level approaches (Oberhardt et al. 2009).

On the contrary, specialized metabolism refers to metabolic pathways that synthesize chemicals not immediately required for the survival of the host organism but, nonetheless, contribute key metabolic traits ensuring the reproductive success of populations (fitness) in complex ecosystems (Fraenkel 1959). Specialized metabolism is found in all living organisms. Notably, specialized metabolic systems have undergone extensive expansion in sessile organisms, including plants, fungi, and bacteria, yielding a tremendous diversity of so-called "secondary metabolites" that far outnumber the currently identified primary metabolites conserved across all three domains of life. Many of these specialized metabolites participate 
A

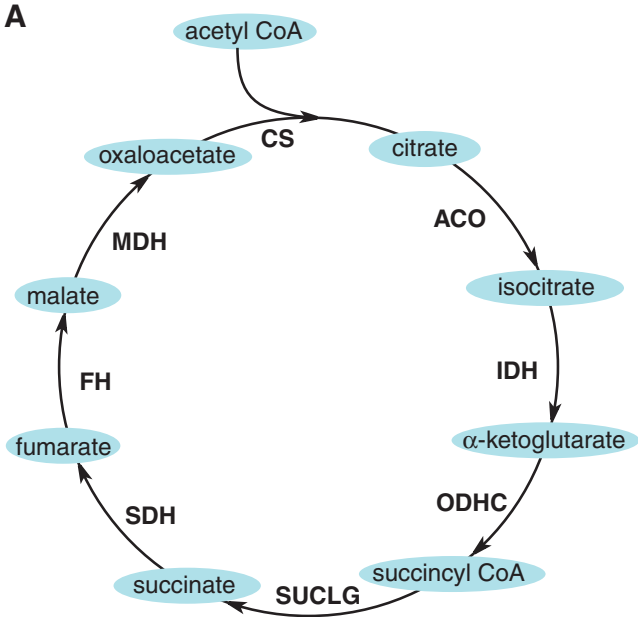

B

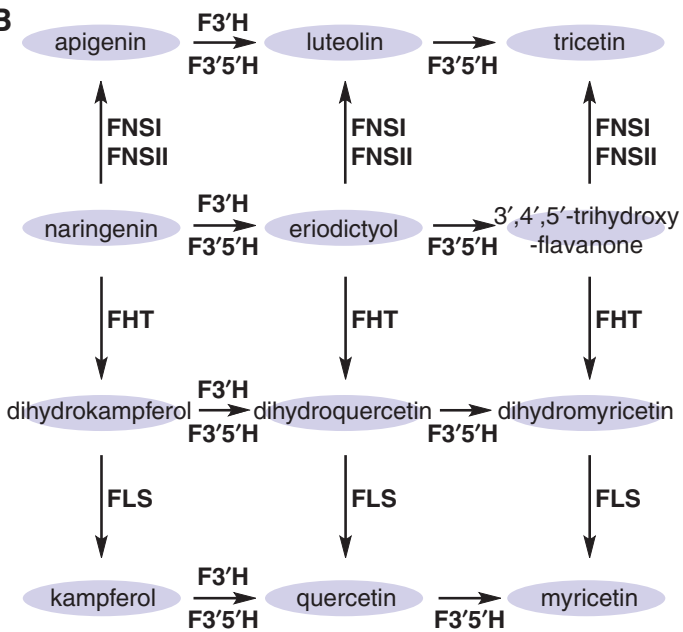

Figure 1. Representative topology of primary and specialized metabolic pathways. $(A)$ The citric acid cycle, central to energy metabolism and conserved among all domains of life, shows a typical linear primary metabolic pathway. (CS) Citrate synthase; (ACO) aconitase; (IDH) isocitrate dehydrogenase; (ODHC) 2-oxoglutarate dehydrogenase complex; (SUCLG) succinate-CoA ligase (GDP-forming); (SDH) succinate dehydrogenase; (FH) fumarate hydratase (or fumarase); (MDH) malate dehydrogenase. (B) The plant flavonoid metabolic pathway represents a metabolic grid commonly seen in specialized metabolism. In a metabolic grid, one metabolic intermediate is often accessed by multiple enzymes and one enzyme often catalyzes parallel reactions on a series of structurally related metabolites. (FNS) Flavone synthase; (FHT) flavanone 3-hydroxylase; (FLS) flavonol synthase; (F3'H) flavonoid 3'-hydroxylase; $\left(\mathrm{F}^{\prime} 5^{\prime} \mathrm{H}\right)$ flavonoid $3^{\prime} 5^{\prime}$-hydroxylases.

in biotic responses such as chemical defenses that inhibit the growth of competing or pathogenic organisms or deterrents against predators (Vicente et al. 2003). Others mediate interspecies communications by color, scent, or more complex small-molecule signaling mechanisms (Gang 2005; Shank and Kolter 2009).

In plants, a number of secondary metabolites have also been recruited to serve as phytohormones that regulate physiological changes in response to specific environmental cues. Finally, many of these metabolites accumulate and provide protection against abiotic stresses including UV radiation, desiccation, and temperature. The evolutionary exploitation of biosynthetic chemical space by rapidly evolving specialized metabolic systems has been a critical adaptive strategy for the sessile lifestyle of plants, fungi, and bacteria that colonize almost every environmental niche of the global ecosystem. The chemicals also likely contributed to the emergence of terrestrial life nearly 500 million years ago (Weng and Chapple 2010).

The tremendous importance of these specialized metabolic pathways to varied life forms and lifestyles is also apparent from their disparate taxonomic distributions. Certain pathways were fixed early in an ancestral lineage and became widespread in all the extant species in that lineage concomitantly with the speciation process, for example, the presence of phenylpropanoid metabolism in all terrestrial plants (Vogt 2010). Some other pathways may only be found in limited genera or even in a single species, for example, penicillin biosynthesis is reported only in a few species of the ascomycetous fungi under the genus Penicillium. Certain specialized metabolic traits have also arisen independently when driven by a strong selective pressure in distantly related species (Pichersky and Lewinsohn 2011). These independent events can occur via similar mechanisms based on orthologous ancestral pathways and enzymes, for example, the multiple emergences of the plant polyketide synthases (PKSs) known as stilbene synthases (STSs) from the ancestral plant PKSs known as chalcone synthases (CHSs) (Pichersky and Lewinsohn 2011). Alternatively, independent emergence of the same chemical trait can occur more rarely from nonorthologous progenitor forms, for example, independent occurrences of gibberellin (GA) biosynthesis in plants and fungi (Tudzynski 2005). The complex phylogenetic distribution pattern seen in specialized metabolism suggests a remarkable degree of metabolic evolvability underpinning these systems that presents us with a rich and expanding natural data set because these pathways arise continuously throughout the evolutionary history of life often over relatively short evolutionary timescales.

Our growing appreciation for the chemical and phylogenetic features of specialized metabolic enzymes highlights some emerging postulates concerning their differing characteristics relative to their "perfected" cousins underpinning primary metabolism. Some of these attributes now being uncovered with quantitative and mechanistic clarity include broad substrate specificity, modest catalytic efficiency, and relaxation of the underlying mechanistic trajectories yielding alternative products (Bar-Even et al. 2011; Nam et al. 2012). Given that ancestral catalytic promiscuity provides an efficient starting point for the evolution of new enzymatic activities under natural selection or neutral drift (O'Brien and Herschlag 1999; Khersonsky and Tawfik 2010), these unconventional catalytic behaviors are arguably major factors underlying evolvability of specialized metabolic systems. Moreover, because specialized metabolic traits are not absolutely 
required for survival, naturally occurring genomic variations that add new modules, subtract existing components, or alter protein activities, lifetimes, and stability often do not cause lethality or drastic fitness loss in individuals inheriting these variations, which further potentiates specialized metabolic evolution.

Naturally occurring specialized metabolic systems are enormously rich and complex evolutionary systems rivaling (if not exceeding) the richness of organismal form traditionally represented in taxonomy (Fig. 1B). Here, we illustrate examples drawn from our current but still limited understanding of some of these systems and discuss the mechanistic basis underlying their origin and evolution at genetic, biochemical, and structural levels. We hope that this account, together with many others addressing the issue of chemodiversity in living systems (Jones et al. 1991; Orians 2000; Pichersky and Gang 2000; Pichersky et al. 2006; Firn and Jones 2009; Weng et al. 2012b), will aid in achieving a coherent and widely applicable framework that explains the exceptional evolvability of enzymes and metabolic systems.

\section{GENOMIC VARIATION AS RAW MATERIAL FOR METABOLIC INNOVATION}

Metabolic pathways, similarly to many biological systems with complex features, adapt by genetic changes in pathway components. Heritable genetic variations, ranging from point mutations, deletions, insertions, gene translocations, and retroposition to chromosome loss, genome rearrangement, and polyploidization, yield the genetically encoded basis for metabolic innovation. Because most plant specialized metabolic pathways do not immediately impact survival, the aforementioned changes can be selectively neutral or only modestly deleterious to the overall fitness of the host individual.

Gene duplication followed by subfunctionalization or neofunctionalization is a major evolutionary mechanism by which new pathways have continually emerged in specialized metabolism (Fig. 2A). In Arabidopsis thaliana, gene duplication of two ancestral P450 genes in the conserved lignin biosynthetic pathway resulted in new alleles that neofunctionalized to catalyze reactions in recently emerged hydroxycinnamoyl spermidine and arabidopyrone biosynthetic pathways (Matsuno et al. 2009; Weng et al. 2012a).

The presence of tandem duplicated genes at adjacent genomic loci is also a common phenomenon underpinning the expansion of specialized metabolic enzyme families in higher eukaryotes (Kliebenstein 2008). Single genes within the tandem pair often show distinct temporal and spatial expression patterns, and more significantly, divergent catalytic properties. For example, the A. thaliana genome contains a tandem cluster harboring five serine carboxypeptidase-like (SCPL) genes on chromosome II (Fraser et al. 2007). Genetic and biochemical analyses of members of this tandem cluster revealed that the encoded SCPL enzymes are sinapoylglucose acyltransferases with overlapping substrate specificities (Fraser et al. 2007). The combined biochemical activities of these five enzymes define the metabolic fingerprints of sinapate esters in Arabidopsis (Fraser et al. 2007). Subsequently, a natural accession of $A$. thaliana, Pna-10, was found to harbor a $13-\mathrm{kb}$ deletion at this sinapate ester locus that eliminates two out of five SCPL genes from this cluster. This gene loss uniquely correlates with a newly discovered sinapate ester profile distinct from the other Arabidopsis accessions surveyed (Li et al. 2010). Interestingly, Pna-17, another A. thaliana accession collected from the same site harboring Pna-10, does not contain this deletion, hinting at unexpected metabolic diversity existing in geographically defined natural populations ( $\mathrm{Li}$ et al. 2010).

The malleability of genomes affords the emergence and evolution of pervasive metabolic operons in bacteria, wherein nonhomologous genes functioning in a particular metabolic pathway are encoded on a continuous stretch of DNA and are often transcribed as one polycistronic messenger RNA (mRNA) (Fig. 2B) (Merino et al. 2008).
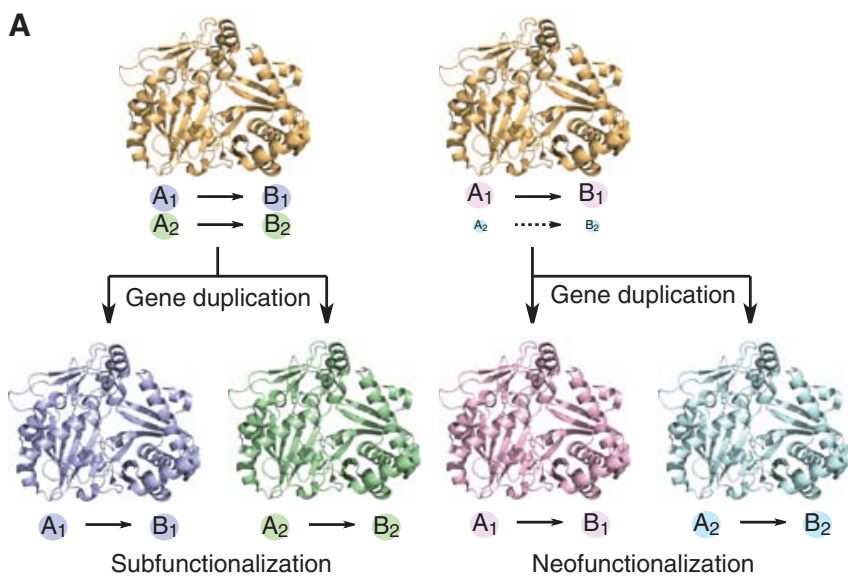

B
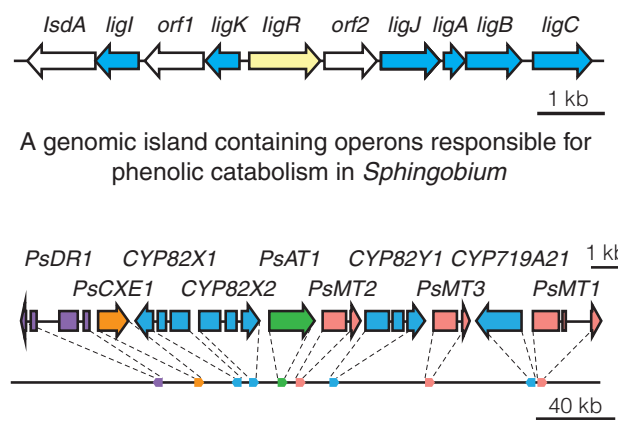

A 10-gene cluster for noscapine biosynthesis in Papaver somniferum

Figure 2. Heritable genomic variations provide the raw material for metabolic innovations in specialized metabolism. $(A)$ Gene duplication followed by subfunctionalization or neofunctionalization is a primary mechanism underpinning divergence in enzyme catalytic functions. (B) Natural selection has driven the prevalent assembly of metabolic operons in bacteria and some operon-like gene clusters underpinning specialized metabolic pathways in plants. 
Comparative genomics and biochemical studies of bacterial metabolism have identified a number of operons encoding diverse specialized metabolic pathways (Barnes et al. 1997; Nishizawa et al. 2000; Penn et al. 2009). Although functionally related genes are commonly dispersed throughout the genome in higher eukaryotes, growing evidence suggests that metabolic evolution in complex organisms may also be driven by the assembly of operonlike gene clusters (Chu et al. 2011). In one remarkable case, the presence of a 10-gene cluster encoding five distinct enzyme classes responsible for specialized alkaloid noscapine biosynthesis was identified in a high-noscapine-producing poppy variety, HN1 (Winzer et al. 2012) (Fig. 2B). Genomic rearrangements that carry genes encoding an advantageous specialized metabolic trait to one genomic locus may be positively selected for the rapid assembly of new specialized pathways (Chu et al. 2011).

Variations in metabolic traits along with genetic heterogeneity are common features of plant populations $(\mathrm{Li}$ et al. 2010; Eckert et al. 2011). Although selfing plants are commonly used as laboratory models, sexual reproduction likely has a substantial role in plant adaptation. Sex accelerates the process of recombining functionally divergent components of specialized metabolic pathways across subpopulations within a more geographically diverse collection of individuals. As a consequence, novel combinations, extreme concentrations of secondary metabolites, or in some cases, metabolites not found in both parents are expressed as a result of sexual reproduction. Indeed, several studies focusing on the impact of hybridization among related plant species on metabolomic profiles in their progeny support this hypothesis (Kirk et al. 2004, 2005, 2012).

\section{EMERGENCE OF RECONFIGURED CATALYTIC MECHANISMS}

Commonly observed features of specialized metabolic systems include the emergence of new catalytic machineries from progenitor enzymes catalyzing alternative reactions or even from noncatalytic proteins. These phenomena reflect a level of dynamic flexibility inherent in the ancestral active site/ligand-binding pocket, affording chemical accommodation of new metabolites. Moreover, the pliability of the overall fold also furnishes access to catalytic mechanisms with only a few mutations, establishing new energetic networks encoding nascent or alternative catalytic mechanisms.

For example, strictosidine synthase (STR), a key enzyme in the biosynthesis of pharmacologically important monoterpenoid indole alkaloids, adopts a six-bladed $\beta$ propeller fold and catalyzes a Pictet-Spengler-type reaction (Ma et al. 2006). STR activity emerged after fixation of mutations encoding a catalytic glutamate involved in amine deprotonation of STR's tyramine substrate. This catalytic residue and mechanism are unrelated to calcium-dependent phosphotriesterase activities found in STR homologs in animals (Fig. 3A) (Ma et al. 2006). In another case, allene oxide synthase (AOS), an atypical cytochrome P450 enzyme in the oxylipin pathway, biosynthesizes the plant defense hormone jasmonic acid. Unlike the majority of P450s, AOS evolved an unusual active site architecture and chemistry using an aromatic $\pi$ system to mediate the precise rearrangement of a fatty acid hydroperoxide substrate into the allene oxide product (Lee et al. 2008).

The $\alpha / \beta$-hydrolase superfamily represents a group of structurally related enzymes and proteins, widely involved in specialized metabolism. In some cases, it is not clear if the protein catalyzes a chemical reaction and in other cases unexpected catalytic mechanisms have repeatedly arisen that substantially deviate from the canonical esterase-lipase function of the $\alpha / \beta$-hydrolase superfamily. In Hevea brasiliensis, hydroxynitrile lyases (HNL), an $\alpha / \beta$-hydrolase fold protein, catalyzes the cleavage of hydroxynitriles into cyanide and aldehydes or ketones as defense mechanisms against herbivores and microbes (Gruber et al. 2004). HNL retains the conventional Ser-His-Asp catalytic triad of $\alpha / \beta$ hydrolases. However, instead of acting as a nucleophile as in
A

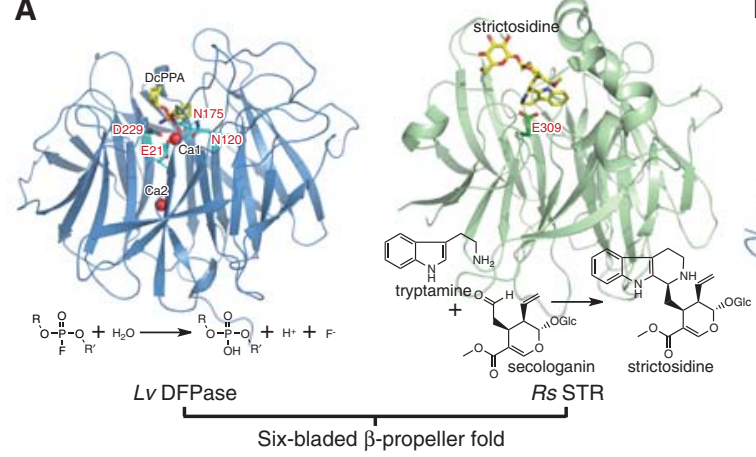

B

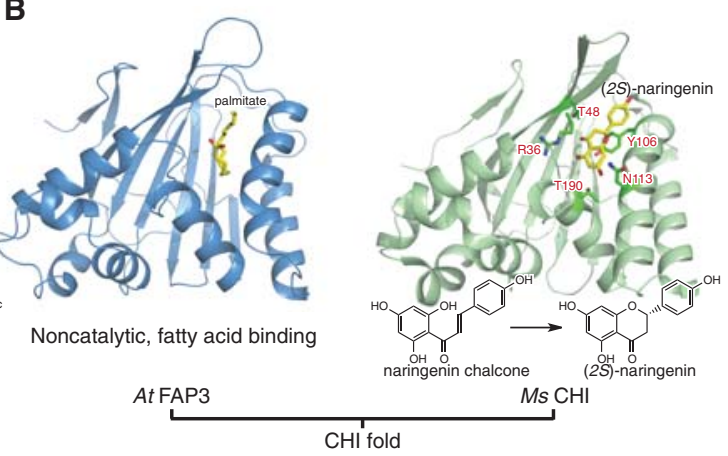

Figure 3. Mechanistic basis for the emergence of new catalytic activities in specialized metabolism. (A) Compared with calciumdependent diisopropylfluorophosphatase from Loligo vulgaris (LvDFPase), the same six-bladed $\beta$-propeller fold in Rauvolfia serpentina evolved a novel catalytic arrangement to function as a strictosidine synthase (RsSTR) during indole alkaloid biosynthesis. (B) Chalcone isomerase (CHI), a key enzyme in plant flavonoid metabolism, evolved from noncatalytic fatty acid binding proteins (FAPs). FAPs are widely distributed in bacteria, fungi, and plants. 
the typical $\alpha / \beta$ hydrolases, the catalytic Ser of HNL functions together with the His and Asp in an alternative general acid/base catalytic mechanism that now recruits an additional active site residue, Lys, to facilitate lyase activity (Gruber et al. 2004). In wild tomato, Solanum habrochaites, an $\alpha / \beta$-hydrolase protein MKS1 neofunctionalized into a 3-ketoacid decarboxylase, a key enzyme in the biosynthetic pathway of methylketone-containing metabolites (Auldridge et al. 2012). MKS1 does not contain a canonical catalytic triad; instead, it carries an Ala-His-Asn triad in place of the Ser-His-Asp triad observed in most $\alpha / \beta$ hydrolases. The His residue, together with a newly emergent active site $\mathrm{Thr}$, uniquely define the efficient decarboxylase activity of MKS1 (Auldridge et al. 2012). In bacteria, members of the $\alpha / \beta$-hydrolase family include enzymes capable of breaking bonds in the biphenyl degradation pathway of Burkholderia xenovorans, as well as in haloalkane dehalogenase and epoxide hydrolase reported in Xanthobacter autotrophicus and Agrobacterium radiobacter respectively (Franken et al. 1991; Nardini et al. 1999). The latter two enzymes contain a nucleophilic Asp in place of the canonical Ser of the $\alpha / \beta$-hydrolase catalytic triad (Franken et al. 1991; Nardini et al. 1999).

In Cannabis sativa, a dimeric $\alpha+\beta$ barrel ferredoxinlike fold protein, olivetolic acid cyclase (OAC), functions as a polyketide cyclase, a previously missing biosynthetic step in the cannabinoid metabolic pathway. OAC catalyzes the $\mathrm{C} 2-\mathrm{C} 7$ intramolecular aldol condensation of a tetraketide intermediate, produced from an upstream type III polyketide synthase (PKS) yielding olivetolic acid (Gagne et al. 2012). Ferredoxin-like fold proteins are widely distributed in all domains of life and are associated with diverse biochemical functions, ranging from monooxygenases in actinorhodin biosynthesis in Streptomyces coelicolor (Sciara et al. 2003) to noncatalytic ribosomal L30 proteins (Mao and Williamson 1999). Interestingly, tetracenomycin F2 cyclase, a polyketide cyclase in the biosynthetic pathway of the antibiotic tetracenomycin C that is produced by various Streptomyces species, also belongs to the ferredoxin-like fold (Thompson et al. 2004), although sequence analysis suggests that the two ferredoxin-like fold polyketide cyclases arose independently in plants and bacteria (Gagne et al. 2012).

In another unexpected discovery, a recent comparative study of chalcone isomerase (CHI) and its structurally related homologs presents a tractable phylogenetic system to study the emergence of novel catalytic functions from nonenzymatic ancestral proteins. $\mathrm{CHI}$ is a stereospecific and diffusion-limited enzyme that catalyzes a key step in plant flavonoid metabolism. Using comparative genomics and biochemical characterization, $\mathrm{CHI}$ was shown to have evolved from a group of fatty-acid-binding proteins (FAPs) also conserved in algal, fungal, and bacterial lineages (Ngaki et al. 2012). FAPs modulate fatty acid content and acyl chain identity in plants, but lack the active site residues necessary for catalyzing the stereospecific Michael-type addition reaction of bona fide $\mathrm{CHI}$ (Fig. 3B) (Ngaki et al. 2012).

\section{THE ROLE OF CATALYTIC PROMISCUITY IN ENZYME EVOLVABILITY}

Proteins are dynamic macromolecules existing not as static sculptures but as a population of marginally stable conformations in solution (Tokuriki and Tawfik 2009b). The relative populations of the various conformational states as well as the energy barriers separating them are modeled as an energy landscape (Henzler-Wildman and Kern 2007). The harmonic and antiharmonic motions of enzymes are likely to be intimately linked to enzyme catalysis, because changing enzyme conformers interact with substrates, transition states, and products during the time course of the catalytic process to overcome significant Pauli repulsion to afford bond making and breaking (Jones 1924; Eisenmesser et al. 2002, 2005; HenzlerWildman et al. 2007). The stochastic nature of enzyme dynamics, likely arising from the limited stability of enzyme folds, also underlies catalytic promiscuity, an enzyme "phenotype" critical to their ongoing evolvability during the process of natural selection. Minor conformational states of an enzyme ensemble likely facilitate the binding of nonnative substrates, mediate alternative reaction coordinates and chemical mechanisms, and/or yield nonnative products (Tokuriki and Tawfik 2009b). Because the population of these minor conformers modulates with varying physicochemical conditions, e.g., $\mathrm{pH}$ and temperature, or through mutations, minor metabolites may form at levels sufficient for natural selection to act on, if they confer a fitness advantage. New mutations that amplify the now beneficial latent activities can then be retained under selection pressure, reshaping the energetic and catalytic landscapes of the evolving enzyme. Neutral drift can also alter the population distribution of conformational states, leading to varying levels of catalytic promiscuity in orthologous enzymes from different species. This catalytic malleability within otherwise orthologous gene products may profoundly impact the functional divergence of these enzymes in a lineage-specific fashion.

It is thought that the demand for high metabolic flux imposes a major selective pressure on the evolution of primary metabolic enzymes (Nam et al. 2012). Because promiscuous substrate binding and/or product formation can negatively impact the catalytic efficiency of an enzyme, catalytic promiscuity was likely attenuated by negative selection during the evolution of primary metabolic systems. Consequently, the extant primary metabolic enzymes that perform conserved core metabolic functions typically show very low levels of catalytic promiscuity and high catalytic efficiencies (Bar-Even et al. 2011).

In contrast, specialized metabolic enzymes evolve under a very different set of evolutionary constraints. These enzymes do not have to provide high metabolic flux but often synthesize metabolites to cope with fluctuating environmental conditions only at the appropriate time. Pressures such as these include situations such as a sudden appearance of particular herbivores or pathogens that can disappear with equal speed, and may only impact subgroups of a reproducing population of individuals. Catalytic promiscuity may even be a selected trait in spe- 
cialized metabolic enzymes, because multifunctional alleles across a population may provide positive advantages under fluctuating environments, becoming fixed in the population at large. Under scenarios such as these, selection for catalytic precision and efficiency, as often seen in primary metabolism, were interrupted in specialized metabolism owing to the need to respond to spatially and temporally fluctuating selective pressures. Such a process of evolution in specialized metabolic enzymes, more often than not, may trade catalytic efficiency for a wider diversity of products capable of enhancing the overall fitness of the organismal population (Yoshikuni et al. 2006; Tokuriki and Tawfik 2009b; Weng et al. $2012 b)$. This view is also consistent with the results generated from those computational approaches attempting to simulate evolutionary processes subjected to rapidly changing selective pressures spread across defined time intervals (Earl and Deem 2004; Kashtan et al. 2007).

Although catalytic promiscuity captures a range of mechanistic processes and outcomes, it can be more precisely described as substrate permissiveness, mechanistic elasticity, and concomitant product diversity (Fig. 4). Each of these specific mechanisms is prevalent in specialized metabolic enzymes. Many enzyme classes are well known for showing high levels of substrate permissiveness, especially those involved in tailoring reactions that decorate and modulate the physiochemical features of core chemical scaffolds, for example, oxidative hydroxylation (Ohnishi et al. 2012), methylation (Kopycki et al. 2008; Huang et al. 2012), acylation (Landmann et al. 2011), glycosidation (Lim et al. 2001), adenylation (Schneider et al. 2005; Westfall et al. 2012), prenylation (Kumano et al. 2010), and redox reactions (Kim et al. 2004; Huang et al. 2009). These enzymes often act on structurally related substrates (Fig. 4A). This feature is key to the establishment of metabolic grids commonly seen in specialized metabolic pathways (Fig. 1B), including the biosynthetic pathways of lignin (Kim et al. 2004; Zhou et al. 2010; Weng et al. 2011), oxylipins (Kienow et al. 2008), and brassinosteroids (Fujioka and Yokota 2003). In comparison, metabolic pathways of primary metabolism are more or less linear, displaying less connectivity (Fig. 1A).

Moreover, substrate permissiveness in some enzyme families facilitated the rapid exploitation of chemical space by parallel tailoring reactions of existing chemical

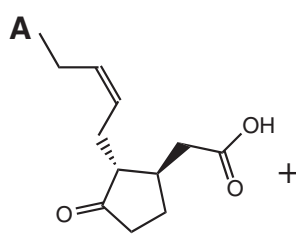

(+)-JA

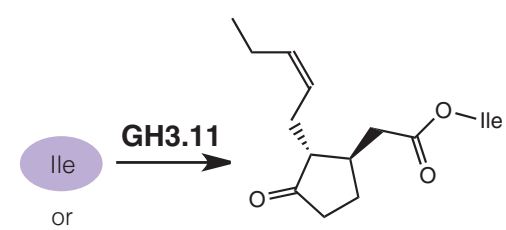

Cys His Tyr Thr Trp

(+)-JA-Ile or

other (+)-JA-amino acid conjugates

B

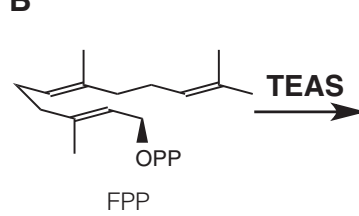

C

(+)-5-epi-aristolochene (79\%)

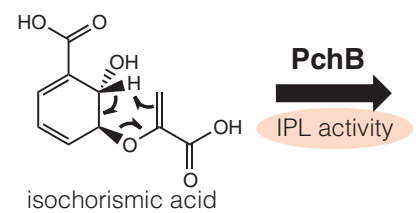

OH

salicylic acid

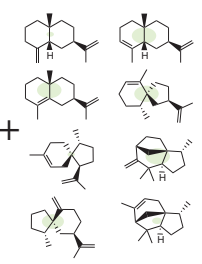

24 minor products $(21 \%)$

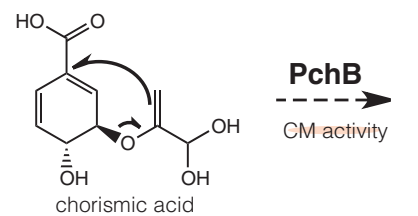

Figure 4. Substrate permissiveness, mechanistic elasticity, and product diversity are three mechanisms contributing to the catalytic promiscuity commonly seen in specialized metabolic enzymes. (A) The Arabidopsis jasmonic acid amino acid conjugating enzyme GH3.11 is capable of using different amino acids as substrates with varying $\mathrm{K}_{\mathrm{M}} \mathrm{s}$. $(B)$ The sesquiterpene synthase tobacco 5-epiaristolochene synthase (TEAS) shows product diversity, producing at least 24 minor products in addition to the major and functionally characterized product 5-epi-aristolochene. $(C)$ The isochorismate-pyruvate lyase from Pseudomonas aeruginosa shows a level of mechanistic elasticity affording latent chorismate mutase activity. 
scaffolds. For example, the plant BAHD acyltransferases often recognize a variety of acyl acceptors, producing multiple esters and amides using the same acyl donor molecule (Landmann et al. 2011). Similarly, the plant GH3 acyl acid amido synthetase family conjugates various amino acids onto the same acyl donor (Fig. 3A) (Westfall et al. 2012). Structural and mutagenic studies have shown that in many cases, the active sites and specific substrate binding pockets associated with substrate permissiveness show conformational flexibility capable of shifting substrate recognition with a very limited number of residue changes (Zubieta et al. 2001; Hrmova et al. 2002; Schneider et al. 2003; Kopycki et al. 2008; Westfall et al. 2012).

Particular families of enzymes often transform the same substrate into multiple products using the same active site machinery, resulting in a diversity of products (Fig. 4B). These protein families include the $\alpha$-helical terpene synthases (TPSs) and the $\alpha \beta \alpha \beta \alpha$ type III PKSs. TPSs use linear prenyl disphosphates as substrates, catalyze loss of pyrophosphate, and often cyclize the resultant carbocations into structurally diverse hydrocarbon products (Chen et al. 2011a). Type III PKSs produce elongated polyketides of varying lengths and tautomeric states, which cyclize by $\mathrm{C}-\mathrm{C}$ and $\mathrm{C}-\mathrm{O}$ bond forming reactions into an array of polyketide-derived secondary compounds (Austin and Noel 2003). TPSs and type III PKSs often produce one major product together with a number of minor products. Moreover, the product distribution profiles of these enzymes are often conditionally sensitive and are exquisitely attuned to minor mutational changes within and around the active site (Austin et al. 2004; O'Maille et al. 2008). Although TPSs and PKSs belong to disparate protein folds, they converge to exploit similar catalytic principles to direct cyclization of their respective highly reactive intermediates. The steric and electronic environment of these dynamic enzyme active sites tames the intrinsic reactivity of the high-energy intermediates by increasing energy barriers separating particular cyclization routes, a mechanism underlying negative catalysis (Rétey 1990).

Catalytic promiscuity can also directly correlate with mechanistic elasticity associated with certain specialized metabolic enzymes (Fig. 4C). For example, isochorismate-pyruvate lyase from $P$. aeruginosa $(\mathrm{PchB})$ synthesizes salicylic acid (SA) and pyruvate from isochorismate for incorporation of the SA molecule into the siderophore pyochelin. Nevertheless, PchB also shows low-level chorismate mutase, activity that converts chorismate to prephenate (Kunzler et al. 2005). Whereas the isochorismate-pyruvate lyase activity of PchB involves the transfer of a hydrogen from $\mathrm{C} 2$ to $\mathrm{C} 9$ with pyruvate elimination, the latent chorismate mutase activity catalyzes $\mathrm{C}-\mathrm{C}$ bond formation between $\mathrm{C} 1$ and $\mathrm{C} 9$ with fission of the $\mathrm{C}-\mathrm{O}$ bond between $\mathrm{C} 3$ and its ether oxygen (Fig. 4C) (Lamb 2011). PchB is structurally related to bacterial chorismate mutase, and its ability to catalyze two different pericyclic reactions arises from an elastic catalytic mechanism, wherein the same enzyme active site stabilizes alternative transition states (Lamb 2011). Notably, the mechanistic elasticity of PchB may also help to uncover the long-sought-after enzyme that catalyzes the conversion of isochorismate to SA in plants by using comparative phylogenetic and biochemical analyses (Dempsey et al. 2011).

\section{HOMEOSTASIS AND DYNAMICS IN SPECIALIZED METABOLISM}

Kinetic attributes, tissue-subcellular localization, dynamic changes in pathway circuitry, in vivo stability, and interactions with other proteins differ from enzyme to enzyme. When integrated into a systems-level model, the additive and nonadditive effects of these characteristics confer emergent catalytic properties key to homeostasis and dynamics of metabolic systems, including specialized metabolism.

Many enzymes show substrate inhibition, in which steady-state catalytic velocity rises and then descends gradually or abruptly as the substrate concentration increases (Reed et al. 2010). Recent studies suggest that substrate inhibition may be a positively selected kinetic property of particular enzymes as a way to fine-tune metabolic flux under varying physiological conditions. Tyrosine hydroxylase $(\mathrm{TH})$, the enzyme catalyzing the first committed step of dopamine biosynthesis in animals, displays strong substrate inhibition (Quinsey et al. 1998). It was later shown that substrate inhibition of $\mathrm{TH}$ ensures a dopamine homeostasis, while cells experience large fluctuations in intracellular tyrosine concentrations owing to diet and tyrosine availability (Fernstrom and Fernstrom 1994; Best et al. 2009). In plant gibberellin (GA) biosynthesis, the first committed step from $(E, E, E$, $)$-geranylgeranyl diphosphate (GGPP) to ent-copalyl diphosphate is catalyzed by ent-copalyl diphosphate synthase (CPS), a diterpene synthase exhibiting substrate inhibition (Prisic and Peters 2007). During seedling de-etiolation, GGPP levels surge abruptly, limiting metabolic overproduction of GA by substrate inhibition. This kinetic rheostat then allows the greater GGPP levels to flow into the biosynthesis of carotenoids, a class of photosynthetic pigments necessary as this developmental process ensues (Fleet and Sun 2005; Prisic and Peters 2007).

Other specialized metabolic enzymes are inhibited by their own products, a kinetic characteristic often tightly linked to varying the resistance to continued metabolic flux control in vivo. For instance, the SA-binding protein 2 (SABP2) from tobacco was initially isolated as a candidate for the SA receptor, because it displays high affinity for SA and is required for the activation of systemic acquired resistance (SARS) in plants (Kumar and Klessig 2003). Genetic, biochemical, and structural studies then revealed that SABP2 is in fact a methylsalicylate (MeSA) esterase, which controls the release of bioactive SA from MeSA to transduce the SARS signal (Forouhar et al. 2005; Park et al. 2007). The potent inhibition of SABP2 by its product SA evolved the ability to quantitatively sense and then modulate the SARS response by control of intracellular SA levels (Forouhar et al. 2005). 
Besides substrate-product inhibition, enzymes are sometimes inhibited by other metabolites present in the cell at the proper location and at the appropriate time. This mechanism has also been related to regulatory mechanisms impacting temporal and spatial control of metabolic flux. For example, PBS3, an acyl acid amido synthetase from Arabidopsis, participates in SA metabolism. PBS3 is capable of conjugating glutamate to four substituted benzoates in vitro (Okrent et al. 2009). Ironically, SA is not a substrate of PBS3, but it effectively inhibits PBS3 activity by binding in a conformation in which the SA carboxylate moiety is positioned distal from the $\alpha$ phosphate of AMP. The $\alpha$ phosphate is the site of carboxyl attack, leading to adenylated carboxyl-bearing intermediates (Westfall et al. 2012). This observation suggests that an SA feedback mechanism exists in PBS3 acutely attuned to the cellular SA levels that are so critical to SARS, SA hormone homeostasis, and SA concentration dynamics during plant defense responses (Okrent et al. 2009).

Specialized metabolic pathways in higher eukaryotes often localize to specific tissue types, specialized cell types, and subcellular compartments. Localization facilitates exploitation of metabolic flux from primary metabolic pathways localized in different organelles and also reshapes the homeostatic characteristics of metabolic pathways otherwise dictated by the intrinsic reactivity of the catalytic and metabolic intermediates together with the kinetic properties of each participating enzyme (Mintz-Oron et al. 2011). For example, the cellular and subcellular localization of enzymes involved in plant alkaloid biosynthesis is remarkably complex (Ziegler and Facchini 2008). In one example, enzymes of a pathway localize differentially in different plant organs or differing cell types of the same organ, fine-tuning the accumulation of specific alkaloids in specific tissues and specialized cell types. On the other hand, the same alkaloid biosynthetic enzymes can be redirected to multiple subcellular compartments, suggesting extensive subcellular trafficking of the pathway intermediates (Ziegler and Facchini 2008).

Cellular-subcellular compartmentalization of specialized metabolic systems is also critical to the specialized functions of many metabolic products in response to biotic and abiotic environmental elicitors. Brassicaceae plants produce glucosinolates as natural deterrents and insecticides against herbivores. Subcompartmentalization of glucosinolates in the form of thioglucosides in vacuoles separates them from the enzyme responsible for their hydrolysis and bioactivation, namely, myrosinase. Myrosinase is packaged as myrosin grains in the vacuoles of particular idioblasts or exists outside the vacuole in the cytosol (Andreasson et al. 2001). As a result of tissue damage by chewing herbivores, myrosinase now comes in contact with its thioglucoside substrates and catalyzes release of thiocyanate or nitrile to deter herbivore feeding (Halkier and Gershenzon 2006).

The seeming "messiness" of specialized metabolic pathways and grids owing to enhanced levels of catalytic promiscuity suggests that the metabolic fluxes in vivo are minimally complex and even potentially reminiscent of chaotic systems (Fig. 1B) (Werndl 2009). Multiple enzymes likely compete for common intermediates, referred to as "hub" metabolites (Fig. 5) (Weng et al. 2012b). Alternatively, the same enzyme may catalyze a series of parallel reactions involving structurally related substrates and products with varying kinetic efficiencies (Fig. 1B). Higher-order metabolons, involving direct physical interactions among enzymes, have also been implicated as key control points in many specialized metabolic pathways, including phenylpropanoid, isoprenoid, alkaloid, and cyanogenic glucoside biosyntheses in plants (Achnine et al. 2004; Weid et al. 2004; Winkel 2004; Jorgensen et al. 2005; Leivar et al. 2005; Nielsen et al. 2008; Chen et al. 2011b). Putative metabolons are postulated to facilitate metabolic channeling, thus exerting a level of kinetic control and specificity by localizing spe-

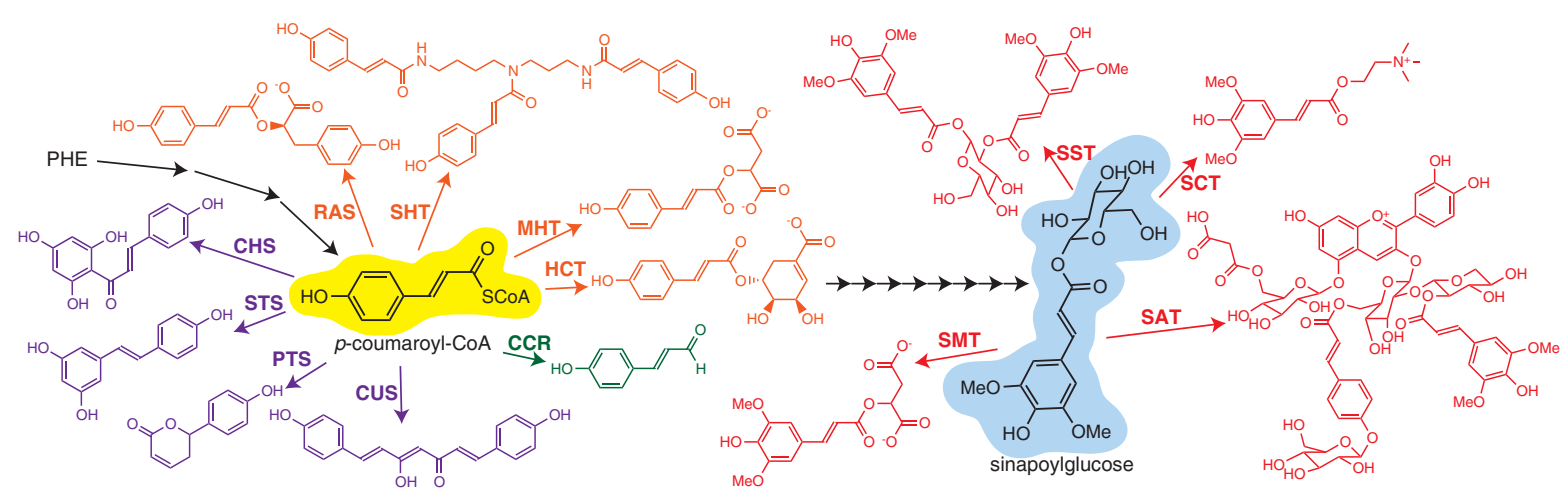

Figure 5. Pathway topology abstracted from plant phenylpropanoid metabolism to illustrate the presence of highly accessed "hub" metabolites, in this case, $p$-coumaroyl-CoA and sinapoylglucose. Orange, purple, green, and red denote reactions catalyzed by BAHD acyltransferases, type III polyketide synthases, NAD(P)H-dependent reductases, and SCPL acyltransferases, respectively. (RAS) Rosmarinic acid synthase; (SHT) spermidine hydroxycinnamoyl transferase; (MHT) malate hydroxycinnamoyl transferase; (HCT) hydroxycinnamoyl-CoA shikimate/quinate hydroxycinnamoyl transferase; (CHS) chalcone synthase; (STS) stilbene synthase; (PTS) psilotin synthase; (CUS) curcumin synthase; (CCR) cinnamoyl-CoA reductase; (SST) sinapoyl-Glc:sinapoyl-Glc sinapoyltransferase; (SCT) sinapoyl-Glc:choline sinapoyltransferase; (SMT) sinapoyl-Glc:malate sinapoyltransferase; (SAT) sinapoyl-Glc:anthocyanin sinapoyltransferase. 
cialized pathways within or on the surface of particular subcellular compartments or by balancing the stoichiometry of participating pathway enzymes. Metabolic channeling may also prevent the premature release of highly reactive or toxic compounds. Although unequivocal demonstration of transient and dynamically changing metabolons continues to elude us, the evolutionary selection of metabolons lends flexibility and order to an otherwise messy and chaotic specialized metabolic system.

\section{FUTURE PERSPECTIVES}

The genetic and selective nature of evolution resulted in clustering of sequence space with the extinction of evolutionary ancestors not present in the extant sequences available for study. However, owing to descent with modification and its historical record encoded in genomes, recent technological advances have facilitated the generation of large-scale ancestral mutant libraries based on phylogenies of extant enzyme sequences (Weinreich et al. 2006; O'Maille et al. 2008; Goldsmith and Tawfik 2012). These developments make it possible to peer into the past with an admittedly slightly out of focus "telescope." Nonetheless, ancestral "resurrections" afford laboratory investigation of the evolutionary trajectories underlying catalytic functional divergences accessible in the historical records of genomes (Lobkovsky et al. 2011).

It has been shown that pervasive epistasis arising from pair-wise residue interactions constrains protein evolution (Bershtein et al. 2006; Halabi et al. 2009; Breen et al. 2012) and also secures evolutionary directionality (Bridgham et al. 2009). However, recent examinations of specialized metabolism show at least anecdotally that these pairwise constraints seem to be relatively relaxed in extant specialized metabolic enzymes, because specific functions of divergent enzymes can often be easily transversed by only a few mutations or even one mutation in some cases (Morita et al. 2007; Huang et al. 2012). Does the enhanced evolvabililty of specialized metabolic enzymes stem from the historical degeneration of the ancestral epistatic networks (Weng et al. 2012b), translating into a broader accessibility of evolutionary trajectories? Comparative analyses of structurally related primary and specialized metabolic enzymes using directed evolution approaches should shed light on this fundamental question in evolutionary biology.

The continual emergence of new pathways in evolving specialized metabolic systems also offers a fascinating experimental framework to examine how complex traits arose by Darwinian processes. By interrogating these phylogenetic mutant libraries representing two or more enzymes catalyzing consecutive steps in a metabolic pathway in an actual host, it is possible to begin probing the evolutionary trajectories underpinning complex metabolic traits with appropriate selections imposed on the hosts. Future efforts will also unravel additional genetic and epigenetic components facilitating the rapid evolution of specialized metabolic systems (Tokuriki and Tawfik 2009a; Dowen et al. 2012).

\section{ACKNOWLEDGMENTS}

This work was supported by grants from the National Science Foundation under Award Nos. EEC-0813570 and MCB-0645794 to J.P.N. J.K.W. is supported by a postdoctoral fellowship from the Pioneer Foundation. J.P.N. is an investigator with the Howard Hughes Medical Institute.

\section{REFERENCES}

Achnine L, Blancaflor EB, Rasmussen S, Dixon RA. 2004. Colocalization of L-phenylalanine ammonia-lyase and cinnamate 4-hydroxylase for metabolic channeling in phenylpropanoid biosynthesis. Plant Cell 16: 3098-3109.

Andreasson E, Bolt Jorgensen L, Hoglund AS, Rask L, Meijer J. 2001. Different myrosinase and idioblast distribution in Arabidopsis and Brassica napus. Plant Physiol 127: 1750-1763.

Auldridge ME, Guo Y, Austin MB, Ramsey J, Fridman E, Pichersky E, Noel JP. 2012. Emergent decarboxylase activity and attenuation of $\alpha / \beta$-hydrolase activity during the evolution of methylketone biosynthesis in tomato. Plant Cell 24: 1596-1607.

Austin MB, Noel JP. 2003. The chalcone synthase superfamily of type III polyketide synthases. Nat Prod Rep 20: 79-110.

Austin MB, Bowman ME, Ferrer JL, Schroder J, Noel JP. 2004. An aldol switch discovered in stilbene synthases mediates cyclization specificity of type III polyketide synthases. Chem Biol 11: 1179-1194.

Bar-Even A, Noor E, Savir Y, Liebermeister W, Davidi D, Tawfik DS, Milo R. 2011. The moderately efficient enzyme: Evolutionary and physicochemical trends shaping enzyme parameters. Biochemistry 50: 4402-4410.

Barnes MR, Duetz WA, Williams PA. 1997. A 3-(3-hydroxyphenyl)propionic acid catabolic pathway in Rhodococcus globerulus PWD1: Cloning and characterization of the hpp operon. J Bacteriol 179: 6145-6153.

Bershtein S, Segal M, Bekerman R, Tokuriki N, Tawfik DS. 2006. Robustness-epistasis link shapes the fitness landscape of a randomly drifting protein. Nature 444: 929-932.

Best JA, Nijhout HF, Reed MC. 2009. Homeostatic mechanisms in dopamine synthesis and release: A mathematical model. Theor Biol Med Model 6: 21.

Breen MS, Kemena C, Vlasov PK, Notredame C, Kondrashov FA. 2012. Epistasis as the primary factor in molecular evolution. Nature 490: 535-538.

Bridgham JT, Ortlund EA, Thornton JW. 2009. An epistatic ratchet constrains the direction of glucocorticoid receptor evolution. Nature 461: 515-519.

Chen F, Tholl D, Bohlmann J, Pichersky E. 2011a. The family of terpene synthases in plants: A mid-size family of genes for specialized metabolism that is highly diversified throughout the kingdom. Plant J 66: 212-229.

Chen HC, Li Q, Shuford CM, Liu J, Muddiman DC, Sederoff RR, Chiang VL. 2011b. Membrane protein complexes catalyze both 4- and 3-hydroxylation of cinnamic acid derivatives in monolignol biosynthesis. Proc Natl Acad Sci 108: $21253-$ 21258.

Chu HY, Wegel E, Osbourn A. 2011. From hormones to secondary metabolism: The emergence of metabolic gene clusters in plants. Plant $J$ 66: 66-79.

Cook PF, Cleland WW. 2007. Enzyme kinetics and mechanism. Garland Science, London; New York.

Dempsey DA, Vlot AC, Wildermuth MC, Klessig DF. 2011. Salicylic acid biosynthesis and metabolism. Arabidopsis Book 9: e0156.

Dowen RH, Pelizzola M, Schmitz RJ, Lister R, Dowen JM, Nery JR, Dixon JE, Ecker JR. 2012. Widespread dynamic DNA methylation in response to biotic stress. Proc Natl Acad Sci 109: E2183-E2191. 
Earl DJ, Deem MW. 2004. Evolvability is a selectable trait. Proc Natl Acad Sci 101: 11531-11536.

Eckert AJ, Wegrzyn JL, Cumbie WP, Goldfarb B, Huber DA, Tolstikov V, Fiehn O, Neale DB. 2011. Association genetics of the loblolly pine (Pinus taeda, Pinaceae) metabolome. New Phytol 193: 890-902.

Eisenmesser EZ, Bosco DA, Akke M, Kern D. 2002. Enzyme dynamics during catalysis. Science 295: 1520-1523.

Eisenmesser EZ, Millet O, Labeikovsky W, Korzhnev DM, Wolf-Watz M, Bosco DA, Skalicky JJ, Kay LE, Kern D. 2005. Intrinsic dynamics of an enzyme underlies catalysis. Nature 438: $117-121$.

Fernstrom JD, Fernstrom MH. 1994. Dietary effects on tyrosine availability and catecholamine synthesis in the central nervous system: Possible relevance to the control of protein intake. Proc Nutr Soc 53: 419-429.

Firn RD, Jones CG. 2009. A Darwinian view of metabolism: Molecular properties determine fitness. J Exp Bot 60: 719726.

Fleet CM, Sun TP. 2005. A DELLAcate balance: The role of gibberellin in plant morphogenesis. Curr Opin Plant Biol 8: $77-85$.

Forouhar F, Yang Y, Kumar D, Chen Y, Fridman E, Park SW, Chiang Y, Acton TB, Montelione GT, Pichersky E, et al. 2005. Structural and biochemical studies identify tobacco SABP2 as a methyl salicylate esterase and implicate it in plant innate immunity. Proc Natl Acad Sci 102: 1773-1778.

Fraenkel GS. 1959. The raison d'etre of secondary plant substances; these odd chemicals arose as a means of protecting plants from insects and now guide insects to food. Science 129: $1466-1470$.

Franken SM, Rozeboom HJ, Kalk KH, Dijkstra BW. 1991. Crystal structure of haloalkane dehalogenase: An enzyme to detoxify halogenated alkanes. EMBO J 10: 1297-1302.

Fraser CM, Thompson MG, Shirley AM, Ralph J, Schoenherr JA, Sinlapadech T, Hall MC, Chapple C. 2007. Related Arabidopsis serine carboxypeptidase-like sinapoylglucose acyltransferases display distinct but overlapping substrate specificities. Plant Physiol 144: 1986-1999.

Fujioka S, Yokota T. 2003. Biosynthesis and metabolism of brassinosteroids. Annu Rev Plant Biol 54: 137-164.

Gagne SJ, Stout JM, Liu E, Boubakir Z, Clark SM, Page JE. 2012. Identification of olivetolic acid cyclase from Cannabis sativa reveals a unique catalytic route to plant polyketides. Proc Natl Acad Sci 109: 12811-12816.

Gang DR. 2005. Evolution of flavors and scents. Annu Rev Plant Biol 56: 301-325.

Goldsmith M, Tawfik DS. 2012. Directed enzyme evolution: Beyond the low-hanging fruit. Curr Opin Struct Biol 22: 406-412.

Gruber K, Gartler G, Krammer B, Schwab H, Kratky C. 2004. Reaction mechanism of hydroxynitrile lyases of the alpha/ $\beta$-hydrolase superfamily: The three-dimensional structure of the transient enzyme-substrate complex certifies the crucial role of LYS236. J Biol Chem 279: 20501-20510.

Halabi N, Rivoire O, Leibler S, Ranganathan R. 2009. Protein sectors: Evolutionary units of three-dimensional structure. Cell 138: 774-786.

Halkier BA, Gershenzon J. 2006. Biology and biochemistry of glucosinolates. Annu Rev Plant Biol 57: 303-333.

Henzler-Wildman K, Kern D. 2007. Dynamic personalities of proteins. Nature 450: 964-972.

Henzler-Wildman KA, Lei M, Thai V, Kerns SJ, Karplus M, Kern D. 2007. A hierarchy of timescales in protein dynamics is linked to enzyme catalysis. Nature 450: 913-916.

Hrmova M, De Gori R, Smith BJ, Fairweather JK, Driguez H, Varghese JN, Fincher GB. 2002. Structural basis for broad substrate specificity in higher plant $\beta$-D-glucan glucohydrolases. Plant Cell 14: 1033-1052.

Huang FC, Horvath G, Molnar P, Turcsi E, Deli J, Schrader J, Sandmann G, Schmidt H, Schwab W. 2009. Substrate promiscuity of RdCCD1, a carotenoid cleavage oxygenase from Rosa damascena. Phytochemistry 70: 457-464.
Huang R, Hippauf F, Rohrbeck D, Haustein M, Wenke K, Feike J, Sorrelle N, Piechulla B, Barkman TJ. 2012. Enzyme functional evolution through improved catalysis of ancestrally nonpreferred substrates. Proc Natl Acad Sci 109: 2966-2971.

Jones JE. 1924. On the determination of molecular fields. II. From the equation of state of a gas. Proc R Soc Lond A 106: $463-477$.

Jones CG, Firn RD, Malcolm SB. 1991. On the evolution of plant secondary chemical diversity [and Discussion]. Philos Trans R Soc Lond B Biol Sci 333: 273-280.

Jorgensen K, Rasmussen AV, Morant M, Nielsen AH, Bjarnholt N, Zagrobelny M, Bak S, Moller BL. 2005. Metabolon formation and metabolic channeling in the biosynthesis of plant natural products. Curr Opin Plant Biol 8: 280-291.

Kashtan N, Noor E, Alon U. 2007. Varying environments can speed up evolution. Proc Natl Acad Sci 104: 13711-13716.

Khersonsky O, Tawfik DS. 2010. Enzyme promiscuity: A mechanistic and evolutionary perspective. Annu Rev Biochem 79: 471-505.

Kienow L, Schneider K, Bartsch M, Stuible HP, Weng H, Miersch O, Wasternack C, Kombrink E. 2008. Jasmonates meet fatty acids: Functional analysis of a new acyl-coenzyme A synthetase family from Arabidopsis thaliana.J Exp Bot 59: 403-419.

Kim SJ, Kim MR, Bedgar DL, Moinuddin SG, Cardenas CL, Davin LB, Kang C, Lewis NG. 2004. Functional reclassification of the putative cinnamyl alcohol dehydrogenase multigene family in Arabidopsis. Proc Natl Acad Sci 101: 14551460 .

Kirk H, Macel M, Klinkhamer PGL, Vrieling K. 2004. Natural hybridization between Senecio jacobaea and Senecio aquaticus: Molecular and chemical evidence. Mol Ecol 13: $2267-$ 2274.

Kirk H, Choi YH, Kim HK, Verpoorte R, van der Meijden E. 2005. Comparing metabolomes: The chemical consequences of hybridization in plants. New Phytol 167: 613-622.

Kirk H, Cheng D, Choi YH, Vrieling K, Klinkhamer PG. 2012. Transgressive segregation of primary and secondary metabolites in $\mathrm{F}_{2}$ hybrids between Jacobaea aquatica and $J$. vulgaris. Metabolomics 8: 211-219.

Kliebenstein DJ. 2008. A role for gene duplication and natural variation of gene expression in the evolution of metabolism. PLoS One 3: e1838.

Kopycki JG, Rauh D, Chumanevich AA, Neumann P, Vogt T, Stubbs MT. 2008. Biochemical and structural analysis of substrate promiscuity in plant $\mathrm{Mg}^{2+}$-dependent O-methyltransferases. J Mol Biol 378: 154-164.

Kühne W. 1877. Über das Verhalten verschiedener organisirter und sog. ungeformter Fermente. Verh naturh-med Ver Heidelb 1: 190-193.

Kumano T, Tomita T, Nishiyama M, Kuzuyama T. 2010. Functional characterization of the promiscuous prenyltransferase responsible for furaquinocin biosynthesis: Identification of a physiological polyketide substrate and its prenylated reaction products. J Biol Chem 285: 39663-39671.

Kumar D, Klessig DF. 2003. High-affinity salicylic acid-binding protein 2 is required for plant innate immunity and has salicylic acid-stimulated lipase activity. Proc Natl Acad Sci 100: $16101-16106$.

Kunzler DE, Sasso S, Gamper M, Hilvert D, Kast P. 2005. Mechanistic insights into the isochorismate pyruvate lyase activity of the catalytically promiscuous $\mathrm{PchB}$ from combinatorial mutagenesis and selection. J Biol Chem 280: $32827-$ 32834.

Lamb AL. 2011. Pericyclic reactions catalyzed by chorismateutilizing enzymes. Biochemistry 50: 7476-7483.

Landmann C, Hucherig S, Fink B, Hoffmann T, Dittlein D, Coiner HA, Schwab W. 2011. Substrate promiscuity of a rosmarinic acid synthase from lavender (Lavandula angustifolia L.). Planta 234: 305-320.

Lee DS, Nioche P, Hamberg M, Raman CS. 2008. Structural insights into the evolutionary paths of oxylipin biosynthetic enzymes. Nature 455: 363-368. 
Leivar P, Gonzalez VM, Castel S, Trelease RN, Lopez-Iglesias C, Arro M, Boronat A, Campos N, Ferrer A, FernandezBusquets X. 2005. Subcellular localization of Arabidopsis 3-hydroxy-3-methylglutaryl-coenzyme A reductase. Plant Physiol 137: 57-69.

Li X, Bergelson J, Chapple C. 2010. The ARABIDOPSIS accession Pna-10 is a naturally occurring sng 1 deletion mutant. Molecular Plant 3: 91-100.

Lim EK, Li Y, Parr A, Jackson R, Ashford DA, Bowles DJ. 2001. Identification of glucosyltransferase genes involved in sinapate metabolism and lignin synthesis in Arabidopsis. $J$ Biol Chem 276: 4344-4349.

Lobkovsky AE, Wolf YI, Koonin EV. 2011. Predictability of evolutionary trajectories in fitness landscapes. PLoS Comput Biol 7: e1002302.

Ma X, Panjikar S, Koepke J, Loris E, Stockigt J. 2006. The structure of Rauvolfia serpentina strictosidine synthase is a novel six-bladed $\beta$-propeller fold in plant proteins. Plant Cell 18: $907-920$.

Mao H, Williamson JR. 1999. Local folding coupled to RNA binding in the yeast ribosomal protein L30. J Mol Biol 292: $345-359$.

Matsuno M, Compagnon V, Schoch GA, Schmitt M, Debayle D, Bassard JE, Pollet B, Hehn A, Heintz D, Ullmann P, et al. 2009. Evolution of a novel phenolic pathway for pollen development. Science 325: $1688-1692$.

Merino E, Jensen RA, Yanofsky C. 2008. Evolution of bacterial trp operons and their regulation. Curr Opin Microbiol 11: $78-86$.

Mintz-Oron S, Meir S, Malitsky S, Ruppin E, Aharoni A, Shlomi T. 2011. Reconstruction of Arabidopsis metabolic network models accounting for subcellular compartmentalization and tissue-specificity. Proc Natl Acad Sci 109: 339-344.

Morita H, Kondo S, Oguro S, Noguchi H, Sugio S, Abe I, Kohno T. 2007. Structural insight into chain-length control and product specificity of pentaketide chromone synthase from Aloe arborescens. Chem Biol 14: 359-369.

Nam H, Lewis NE, Lerman JA, Lee DH, Chang RL, Kim D, Palsson BO. 2012. Network context and selection in the evolution to enzyme specificity. Science 337: 1101-1104.

Nardini M, Ridder IS, Rozeboom HJ, Kalk KH, Rink R, Janssen DB, Dijkstra BW. 1999. The x-ray structure of epoxide hydrolase from Agrobacterium radiobacter AD1. An enzyme to detoxify harmful epoxides. J Biol Chem 274: 1457914586

Ngaki MN, Louie GV, Philippe RN, Manning G, Pojer F, Bowman ME, Li L, Larsen E, Wurtele ES, Noel JP. 2012. Evolution of the chalcone-isomerase fold from fatty-acid binding to stereospecific catalysis. Nature 485: 530-533.

Nielsen KA, Tattersall DB, Jones PR, Moller BL. 2008. Metabolon formation in dhurrin biosynthesis. Phytochemistry 69: $88-98$.

Nishizawa T, Ueda A, Asayama M, Fujii K, Harada K, Ochi K, Shirai M. 2000. Polyketide synthase gene coupled to the peptide synthetase module involved in the biosynthesis of the cyclic heptapeptide microcystin. J Biochem 127: 779789.

Oberhardt MA, Palsson BO, Papin JA. 2009. Applications of genome-scale metabolic reconstructions. Mol Syst Biol 5: 320 .

O'Brien PJ, Herschlag D. 1999. Catalytic promiscuity and the evolution of new enzymatic activities. Chem Biol 6: R91R105.

Ohnishi T, Godza B, Watanabe B, Fujioka S, Hategan L, Ide K, Shibata K, Yokota T, Szekeres M, Mizutani M. 2012. CYP90A1/CPD, a Brassinosteroid biosynthetic cytochrome P450 of Arabidopsis, catalyzes C-3 oxidation. J Biol Chem 287: $31551-31560$

Okrent RA, Brooks MD, Wildermuth MC. 2009. Arabidopsis GH3.12 (PBS3) conjugates amino acids to 4-substituted benzoates and is inhibited by salicylate. J Biol Chem 284: 9742-9754.

O’Maille PE, Malone A, Dellas N, Andes Hess BJr, Smentek L, Sheehan I, Greenhagen BT, Chappell J, Manning G, Noel JP.
2008. Quantitative exploration of the catalytic landscape separating divergent plant sesquiterpene synthases. Nat Chem Biol 4: 617-623.

Orians CM. 2000. The effects of hybridization in plants on secondary chemistry: Implications for the ecology and evolution of plant-herbivore interactions. Am J Bot 87: 17491756.

Park SW, Kaimoyo E, Kumar D, Mosher S, Klessig DF. 2007. Methyl salicylate is a critical mobile signal for plant systemic acquired resistance. Science 318: 113-116.

Penn K, Jenkins C, Nett M, Udwary DW, Gontang EA, McGlinchey RP, Foster B, Lapidus A, Podell S, Allen EE, et al. 2009. Genomic islands link secondary metabolism to functional adaptation in marine Actinobacteria. ISME J 3: 1193-1203.

Pichersky E, Gang DR. 2000. Genetics and biochemistry of secondary metabolites in plants: An evolutionary perspective. Trends Plant Sci 5: 439-445.

Pichersky E, Lewinsohn E. 2011. Convergent evolution in plant specialized metabolism. Annu Rev Plant Biol 62: 549-566.

Pichersky E, Noel JP, Dudareva N. 2006. Biosynthesis of plant volatiles: Nature's diversity and ingenuity. Science 311: $808-811$

Prisic S, Peters RJ. 2007. Synergistic substrate inhibition of ent-copalyl diphosphate synthase: A potential feed-forward inhibition mechanism limiting gibberellin metabolism. Plant Physiol 144: 445-454.

Quinsey NS, Luong AQ, Dickson PW. 1998. Mutational analysis of substrate inhibition in tyrosine hydroxylase. $J$ Neurochem 71: 2132-2138.

Reed MC, Lieb A, Nijhout HF. 2010. The biological significance of substrate inhibition: A mechanism with diverse functions. Bioessays 32: 422-429.

Rétey J. 1990. Enzymic reaction selectivity by negative catalysis or how do enzymes deal with highly reactive intermediates? Angew Chem Int Ed Engl 29: 355-361.

Schneider K, Hovel K, Witzel K, Hamberger B, Schomburg D, Kombrink E, Stuible HP. 2003. The substrate specificity-determining amino acid code of 4-coumarate:CoA ligase. Proc Natl Acad Sci 100: 8601-8606.

Schneider K, Kienow L, Schmelzer E, Colby T, Bartsch M, Miersch O, Wasternack C, Kombrink E, Stuible HP. 2005. A new type of peroxisomal acyl-coenzyme A synthetase from Arabidopsis thaliana has the catalytic capacity to activate biosynthetic precursors of jasmonic acid. J Biol Chem 280: $13962-13972$

Sciara G, Kendrew SG, Miele AE, Marsh NG, Federici L, Malatesta F, Schimperna G, Savino C, Vallone B. 2003. The structure of ActVA-Orf6, a novel type of monooxygenase involved in actinorhodin biosynthesis. EMBO J 22: 205-215.

Shank EA, Kolter R. 2009. New developments in microbial interspecies signaling. Curr Opin Microbiol 12: 205-214.

Shevelev IV, Hubscher U. 2002. The 3' $5^{\prime}$ exonucleases. Nat Rev Mol Cell Biol 3: 364-376.

Thompson TB, Katayama K, Watanabe K, Hutchinson CR, Rayment I. 2004. Structural and functional analysis of tetracenomycin F2 cyclase from Streptomyces glaucescens. A type II polyketide cyclase. J Biol Chem 279: 37956-37963.

Tokuriki N, Tawfik DS. 2009a. Chaperonin overexpression promotes genetic variation and enzyme evolution. Nature 459: $668-673$

Tokuriki N, Tawfik DS. 2009b. Protein dynamism and evolvability. Science 324: 203-207.

Tudzynski B. 2005. Gibberellin biosynthesis in fungi: Genes, enzymes, evolution, and impact on biotechnology. Appl Microbiol Biotechnol 66: 597-611.

Vicente MF, Basilio A, Cabello A, Pelaez F. 2003. Microbial natural products as a source of antifungals. Clin Microbiol Infect 9: 15-32.

Vogt T. 2010. Phenylpropanoid biosynthesis. Mol Plant 3: 2 20.

Weid M, Ziegler J, Kutchan TM. 2004. The roles of latex and the vascular bundle in morphine biosynthesis in the opium poppy, Papaver somniferum. Proc Natl Acad Sci 101: 13957-13962. 
Weinreich DM, Delaney NF, Depristo MA, Hartl DL. 2006. Darwinian evolution can follow only very few mutational paths to fitter proteins. Science 312: 111-114.

Weng JK, Chapple C. 2010. The origin and evolution of lignin biosynthesis. New Phytol 187: 273-285.

Weng JK, Akiyama T, Ralph J, Chapple C. 2011. Independent recruitment of an O-methyltransferase for syringyl lignin biosynthesis in Selaginella moellendorffii. Plant Cell 23: $2708-2724$.

Weng JK, Li Y, Mo H, Chapple C. 2012a. Assembly of an evolutionarily new pathway for $\alpha$-pyrone biosynthesis in Arabidopsis. Science 337: 960-964.

Weng JK, Philippe RN, Noel JP. 2012b. The rise of chemodiversity in plants. Science 336: $1667-1670$.

Werndl C. 2009. What are the new implications of chaos for unpredictability? Br J Philos Sci 60: 195-220.

Westfall CS, Zubieta C, Herrmann J, Kapp U, Nanao MH, Jez JM. 2012. Structural basis for prereceptor modulation of plant hormones by GH3 proteins. Science 336: 1708-1711.
Winkel BS. 2004. Metabolic channeling in plants. Annu Rev Plant Biol 55: 85-107.

Winzer T, Gazda V, He Z, Kaminski F, Kern M, Larson TR, Li Y, Meade F, Teodor R, Vaistij FE, et al. 2012. A Papaver somniferum 10-gene cluster for synthesis of the anticancer alkaloid noscapine. Science 336: 1704-1708.

Yoshikuni Y, Ferrin TE, Keasling JD. 2006. Designed divergent evolution of enzyme function. Nature 440: 10781082.

Zhou R, Jackson L, Shadle G, Nakashima J, Temple S, Chen F, Dixon RA. 2010. Distinct cinnamoyl CoA reductases involved in parallel routes to lignin in Medicago truncatula. Proc Natl Acad Sci 107: 17803-17808.

Ziegler J, Facchini PJ. 2008. Alkaloid biosynthesis: Metabolism and trafficking. Annu Rev Plant Biol 59: 735-769.

Zubieta C, He XZ, Dixon RA, Noel JP. 2001. Structures of two natural product methyltransferases reveal the basis for substrate specificity in plant O-methyltransferases. Nat Struct Biol 8: 271-279. 


\section{$\$_{\text {CSH }}^{\infty}$ Cold Spring Harbor Symposia SYMPOSIA}

\section{The Remarkable Pliability and Promiscuity of Specialized Metabolism}

J.-K. Weng and J.P. Noel

Cold Spring Harb Symp Quant Biol 2012 77: 309-320 originally published online December 26, 2012

Access the most recent version at doi:10.1101/sqb.2012.77.014787

References This article cites 113 articles, 46 of which can be accessed free at: http://symposium.cshlp.org/content/77/309.full.html\#ref-list-1

\section{License}

Email Alerting Receive free email alerts when new articles cite this article - sign up in Service the box at the top right corner of the article or click here. 